\title{
Spitalleben in der Aufklärung
}

\author{
Von Urs Boschung
}

Das Krankenhaus, von dem hier die Rede ist ${ }^{1}$, wurde 1729 in Edinburg eröffnet, nachdem einige Philanthropen, unter ihnen Alexander Monro I, mit der Hilfe von 352 Subskribenten die nötigen Mittel zusammengebracht hatten. Edinburg ist die erste derartige Anstalt außerhalb von London, wo sich 1720 mit der Westminster Infirmary und 1726 mit Guy's Hospital (und später weiteren Gründungen) eine eigentliche «alliance against misery» gebildet hatte, indem vermögende Kreise sich verpflichteten, regelmäßig Geldbeträge einzubezahlen, um so für kranke Arme medizinische Hilfe bereitzustellen. Christliche Barmherzigkeit und merkantilistisches Nützlichkeitsdenken fanden ein weites Betätigungsfeld, hatte doch der Londoner Quäker und Kaufmann John Bellers 1714 berechnet, daß das Königreich jährlich hunderttausend Einwohner verlor allein durch mangelhafte medizinische Betreuung.

Die Anfänge der Edinburger Infirmary waren bescheiden. Die Zahl der Manager, die im Auftrag der Subskribenten die Anstalt leiteten, der Ärzte, die turnusgemäß den medizinischen Dienst versahen, und des Hilfspersonals überstieg noch die Zahl der Patienten (1729: 35). Bald jedoch erfolgte ein bedeutender Aufschwung. 1736 traf die königliche Anerkennung ein, so daß der offizielle Name nun lautete: Royal Infirmary of Edinburgh. Von 1738 bis 1748 entstand ein neues, repräsentatives Gebäude mit U-förmigem Grundriß, in dem auf vier Stockwerken maximal 228 Patienten untergebracht werden konnten (und in dem noch Lister wirkte, es bestand bis 1879). Die Zahl der aufgenommenen Patienten entwickelte sich wie folgt: 1760: 840; 1770: 1302; 1780: 2268; 1790: 1896; 1800: 2005. 1750 wurden zum Ersatz des bisherigen Rotationssystems erstmals zwei Ärzte durch das Spital fest angestellt, 1769 kamen dazu noch vier Chirurgen. Finanziert wurde das Spital in späteren Jahren nur noch zum kleinen Teil durch Beiträge der Subskribenten, viel mehr durch den Ertrag von Schenkungen und Grund-

${ }^{1}$ Guenter B. Risse, Hospital life in Enlightenment Scotland. Care and teaching at the Royal Infirmary of Edinburgh (Cambridge History of Medicine), Cambridge, University Press, 1986; XI, 450 S., ill. 
stücken, durch Beiträge der Armee und der Marine, durch Sammlungen und - bis zu einem Viertel der Einnahmen - durch den Verkauf von Eintrittsbilletten an Medizinstudenten und Chirurgenlehrlinge, die seit der Eröffnung die Anstalt in steigendem Masse besuchten und von 1748 an formellen klinischen Unterricht erhielten.

Professor Risses Buch konzentriert sich in seinem Hauptteil auf den Zeitraum 1770-1800, dies aus Gründen der Quellenlage, die für den genannten Zeitraum ganz ungewöhnlich gut ist. Neben den Unterlagen der Spitaladministration (Aufnahmeregister der Patienten, Protokolle der Managersitzungen usw.) sind medizinische Journale der Abteilungen und 14 von Studenten geführte Nachschriften des klinischen Unterrichts mit insgesamt 808 ausführlichen Fallaufzeichnungen und den Vorlesungen der Professoren erhalten. Diese ungemein reichhaltigen Dokumente erlauben es, das Spitalleben dreier Dekaden in allen Einzelheiten und mit allen wünschbaren statistischen Belegen nachzuzeichnen, sowohl für die Patienten (Herkunft, Art der Leiden, Verlauf) wie auch für die Mediziner und den Stand ihrer Kunst und den Studentenunterricht, für den Edinburg berühmt war. Aus der Fülle der bemerkenswerten Einzeltatsachen kann hier nur weniges erwähnt werden. Um die Krankheitsverteilung zu ermitteln, wurde aus total etwa 52000 Patienten eine repräsentative Stichprobe von 3047 Fällen gewonnen; im Aufnahmeregister wurde von 1770 bis 1800 in jedem fünften Jahr jede vierte Seite ausgezählt. Die Diagnosen verteilen sich wie folgt: Harnwegs- und Geschlechtskrankheiten $20 \%$, Infektionskrankheiten $15 \%$, chirurgische Infektionskrankheiten $11 \%$, Krankheiten der Atemwege $11 \%$ usw.; zu $71 \%$ handelt es sich um medizinische, zu $20 \%$ um chirurgische Leiden. In diesen wie in den folgenden Zahlen widerspiegelt sich die Verteilung von Krankheiten in der Bevölkerung, aber ebensosehr die Aufnahmepolitik des Spitals. Die Krankheitsgruppen werden von Professor Risse ausführlich einzeln erörtert, wobei jedesmal angegeben wird, was darunter in der zeitgenössischen Terminologie subsumiert wird. Die Hospitalisationsdauer betrug durchschnittlich 31,3 Tage, das Durchschnittsalter bei den Männern 32, bei den Frauen 25 Jahre, die durchschnittliche Gesamtmortalität 4\% (bei den 808 klinischen Fällen 7,8\%).

Ebenso eingehend kommt die Therapie zur Sprache, die mit Ausnahme der Anwendung der Elektrizität weitgehend in traditionellen Bahnen verläuft. Von nicht zu unterschätzender Wirkung für die kranken Armen muß allein der Aufenthalt in geheizten, gut gelüfteten, sauberen Abteilungen und die regelmäßige Ernährung gewesen sein. Anhand der 808 klini- 
schen Fälle läßt sich belegen, daß die am häufigsten verwendeten Arzneimittelgruppen die Abführmittel (bei $26 \%$ der Fälle) und die Schmerzmittel $(16 \%)$ waren. Bei $25 \%$ der Patienten wurde Blut entzogen (bei $14 \%$ in Form des Aderlasses), bei $23 \%$ Blasenpflaster angelegt, bei $16 \%$ wurden Umschläge verordnet, bei $11 \%$ Bäder. Sogar die Menge des gelassenen Blutes läßt sich statistisch, nach den behandelnden Ärzten geordnet, in eine Tabelle bringen; die Mengen reichen von 9,8 bis 25,8 Unzen (300-750g) im Durchschnitt pro Krankheitsfall. Alle derartigen Zusammenstellungen werden von Professor Risse vor dem Hintergrund der zeitgenössischen Literatur aus Edinburg und anderswo kommentiert und ins rechte Licht gerückt.

Seit der Eröffnung 1729 bestand nach dem Vorbild von Leiden eine enge Verbindung zwischen dem Edinburger Spital und der (1726 errichteten) Medizinischen Fakultät. Anfangs begleiteten die Studenten die behandelnden Ärzte und Chirurgen; die Spitalverwaltung verkaufte 1738 hundert Zulassungen zu zwei Guineen. 1748 nahm der Professor der praktischen Medizin John Rutherford den klinischen Unterricht auf; von 1750 an stand ihm dafür eine eigene klinische Abteilung zur Verfügung, für die er sich die Patienten im Spital aussuchte; anfänglich waren es 10, 1790100 Betten. Neben bzw. nach Rutherford wirkten als Professoren Alexander Monro II und Robert Whytt, in der von Professor Risse untersuchten Periode William Cullen, John und James Gregory, um nur die bekanntesten Namen zu nennen. Der Vergleich der Veröffentlichungen dieser Ärzte mit den Vorlesungsnachschriften und den Fallberichten läßt den Schluß zu, daß die Theorie, z. B. Cullens neuropathologisches System, zwar für die nosologische Klassifizierung der beobachteten Krankheiten angewandt wurde, in der Praxis jedoch vorsichtige Empirie mit gelinder Unterstützung natürlicher Heilvorgänge maßgebend war. Auch die pathologische Anatomie spielte eine geringe Rolle; von den 57 auf der Unterrichtsabteilung zwischen 1770 bis 1800 verstorbenen Patienten wurden 30 obduziert; von den 15 aus den übrigen Abteilungen 6. So ist es auch nicht sehr verwunderlich zu sehen, daß die Chirurgie und Geburtshilfe und deren Vertreter im Spital wie im Unterricht eine schwache Stellung einnahmen und sich der Medizin und den Medizinern unterzuordnen hatten.

Der klinische Unterricht in Edinburg galt im letzten Drittel des 18. Jahrhunderts als einer der besten in Europa, nicht zuletzt dank dem Einsatz der Studenten, die sich wiederholt gegen verschiedene Einschränkungen durch die auf die Schonung der Patienten bedachte Verwaltung zur Wehr gesetzt 
hatten. Zwar übernahmen die Studenten nicht wie in Pavia (von Tissot geplant und von Johann Peter Frank verwirklicht) selbst Verantwortung für den Patienten. Dennoch hatten sie reichlich Gelegenheit, eine Vielzahl von Kranken zu beobachten, Verläufe kennenzulernen, sich in Befragung und Besichtigung der Patienten zu üben, an den diagnostischen und therapeutischen Überlegungen der Ärzte teilzunehmen und nicht zuletzt jenes Mitgefühl (sympathy) mit den kranken Mitmenschen zu empfinden, auf das in Edinburg so sehr Wert gelegt wurde (z. B. von John Gregory). Schließlich sollte - daran war den klinischen Lehrern, z. B. Cullen, ebenso gelegen - das kritische Denken der Zuhörer geweckt werden.

Professor Risses Buch beeindruckt nicht nur durch die Fülle seiner Einzelheiten, sondern ebenso sehr durch die souveräne Meisterung des Stoffes, der in logischer Abfolge mit Eleganz und Witz gestaltet ist. In den Anmerkungen und im Anhang finden sich ergänzende Angaben, u. a. zehn Fallbeschreibungen im Wortlaut, ein Kapitel über die Arzneimitteltherapie, verfaßt von J. Worth Estes, und eine reiche Literatur, schließlich ein Register.

Wer bisher in Verallgemeinerung des Berichts von Tenon über das Pariser Hôtel-Dieu die Spitäler des 18. Jahrhunderts als «Todesfallen» betrachtet hat, wird in Zukunft differenzierter urteilen müssen. Immerhin verließen (auf Grund der Stichprobe von 3047 Fällen und bei allen von Professor Risse eingehend diskutierten Einschränkungen) $80 \%$ der Patienten das Spital «geheilt» oder «gebessert». Wenn auch die Volksgesundheit Britanniens dadurch nicht entscheidend beeinflußt wurde, so bedeutet dies doch in dreißig Jahren für etwa 40000 Menschen die Meisterung einer existenzbedrohenden Krise.

Prof. Urs Boschung

Medizinhistorisches Institut

Bühlstraße 26

CH-3012 Bern 